\title{
ASTRONOMICAL EFFECTS OF CURRENT CHANGES IN FUNDAMENTAL ASTROMETRIC REFERENCES
}

\author{
J. KOVALEVSKY \\ OCA/CERGA - UMR/CNRS 6527 \\ Av. Copernic, F 06130-Grasse, France \\ AND \\ D. MCCARTHY \\ U.S. Naval Observatory \\ 3450 Massachusetts Ave, Washington DC 20390, USA
}

\begin{abstract}
We present a first description of the changes in the current procedures that will have to be performed in order to take into account the adoption of the ICRS as the new reference system and new nutation-precession series. A number of choices will have to be made: they are presented in this paper.
\end{abstract}

\section{Introduction}

Although this Joint Discussion concerns the specific problem of a new nutation model associated with an improved constant of precession, it is not possible to separate these changes from the simultaneous changes occurring with the adoption of the extragalactic reference system ICRS and its realizations in radio as well as in visual wavelengths (ICRF and Hipparcos catalogue). At this time, there is a fundamental change in the concepts, the consequences of which have not yet been fully analyzed, and this paper is a contribution to an intellectual effort that should be undertaken in the next few years to establish new procedures that astronomers will have to adopt to reduce astrometric observations.

Starting from the beginning of the 21st century, the situation will be the following:

- The reference system to which all positions and motions should be referred will be the International Celestial Reference System (ICRS) (Ma and Feissel, 1997) which is based upon the assumption that positions of distant extragalactic objects have no global rotation. The corresponding coordinate axes will be fixed in space and close to the FK5 J2000 equator and equinox within the intrinsic errors of the latter.

- The new nutation series will be given in the new reference system.

- The precession formulae will consist of the additional terms to be added to the nutation in order to describe the motion of the celestial pole in the ICRS.

- The complicated situation which arises from the different realizations of the equinox (dynamical or FK5 catalog equinox) will disappear, and the equinox will be replaced by the single fixed origin of the International Celestial Reference Frame (ICRF).

Let us comment upon the practical consequences of these changes. 


\section{Change of fundamental catalog}

The transformation from the FK5 catalog to Hipparcos cannot be reduced to a couple of rotations, one at epoch and another proportional to time. At the level of accuracy that is reached, the regional errors of FK5 are significant and, depending upon how they are modeled, the result of the rotations are different. The transformation formulae must also include some developments as functions of right ascension and declination to be applied to the coordinates of FK5 in order to obtain them in the ICRS. However, such a work remains to be finalized. The difficulty encountered is that the results depend significantly with the choice of the stars entering into the comparison. In particular, double stars and stars presenting a non-uniform proper motion should be excluded. Some stars may even not have been identified as such, but the proper motion in FK5, obtained from observations over several decades, may be different from the quasi-instantaneous proper motion obtained by Hipparcos, which may include some effect due to an orbital motion smoothed out in the FK5.

In any case, even if, despite considerable regional effects, one is looking for only the global orientation and spin differences between Hipparcos and FK5 catalogs, one must introduce in their determination, some representation of regional errors. This is what was done by Mignard and Froeschlé and the result is published in Volume 3 of the catalogue (ESA 1997, Section 19.2), with non-linear effects computed but not considered as sufficiently significant yet to be published.

The results are the following:

1 - Orientation of the FK5 in the Hipparcos system referred to ICRS

$$
\begin{aligned}
& \varepsilon_{x}=-18.8 \pm 2.3 \mathrm{mas} \\
& \varepsilon_{y}=-12.3 \pm 2.3 \mathrm{mas} \\
& \varepsilon_{z}=+16.8 \pm 2.3 \mathrm{mas}
\end{aligned}
$$

The transformation matrix from FK5 system to Hipparcos is

$$
R_{\varepsilon}=\left(\begin{array}{ccc}
1 & \varepsilon_{\mathrm{x}} & -\varepsilon_{\mathrm{y}} \\
-\varepsilon_{\mathrm{x}} & 1 & \varepsilon_{\mathrm{z}} \\
\varepsilon_{\mathrm{y}} & -\varepsilon_{\mathrm{z}} & 1
\end{array}\right)
$$

2 - Spin of the FK5 system in the Hipparcos system referred to ICRS

$$
\begin{aligned}
& \omega_{x}=-0.10 \pm 0.10 \mathrm{mas} / \text { year } \\
& \omega_{y}=+0.43 \pm 0.10 \mathrm{mas} / \text { year } \\
& \omega_{z}=+0.88 \pm 0.10 \mathrm{mas} / \text { year }
\end{aligned}
$$

The rotation matrix $R_{\omega}$ is similarly at time $t$ :

$$
R_{\omega}=\left(\begin{array}{ccc}
0 & \omega_{\mathrm{x}} & -\omega_{\mathrm{y}} \\
-\omega_{\mathrm{x}} & 0 & \omega_{\mathrm{z}} \\
\omega_{\mathrm{y}} & -\omega_{\mathrm{z}} & 0
\end{array}\right)(t-1991.25)
$$

It is useful, at this point, to remind that the Hipparcos system is the ICRS with an uncertainty of $\sigma_{\varepsilon}= \pm 0.60$ mas at epoch (1991.25) and $\sigma_{\omega}= \pm 0.25 \mathrm{mas} / \mathrm{year}$ in spin.

It is to be noted, that the preceding values concern only the systems of coordinates. There are a large regional differences, which are in some cases larger than 100 mas in position and 4 mas/year in proper motions, so that these formulae should not be used to correct the positions of individual stars.

Similar formulae can be derived for the epoch J2000 although they will be less precise, due to the time degradation of the Hipparcos catalog and of the FK5. Similar techniques should be applied to other catalogs based upon the FK5, taking into account, when necessary, the formulae describing the difference between regional errors of the FK5 and the other catalog. For instance, for the PPM Catalogue, the results are the following (ESA 1997, Section 19.3):

$$
\varepsilon_{x}=-22.5 \pm 2.4 \mathrm{mas}
$$




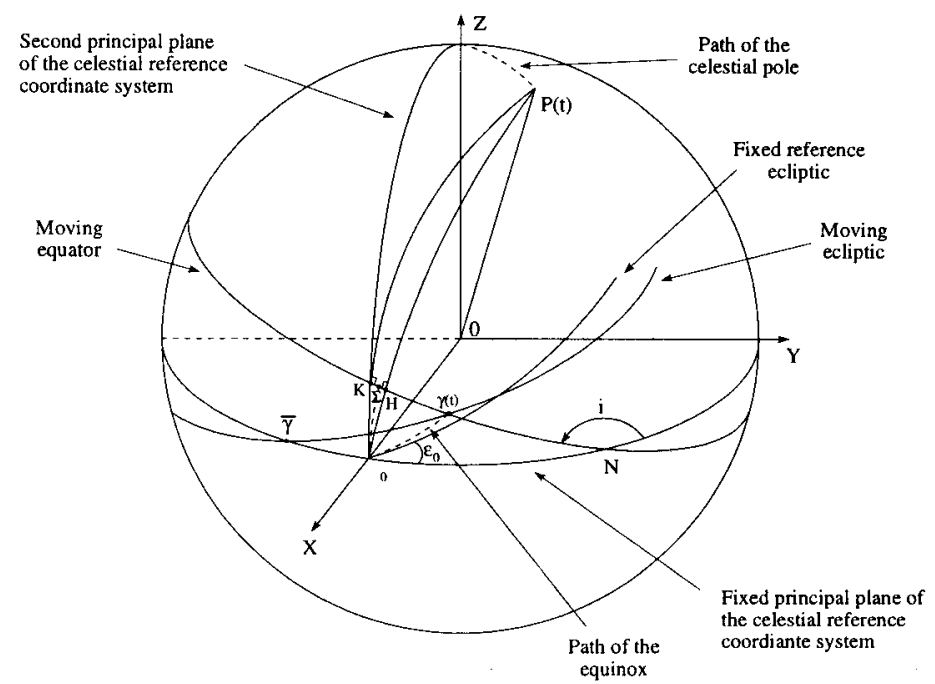

Figure 1. Possible origins of the equatorial frame. For the clarity of the figure, $t<0$.

$$
\begin{gathered}
\varepsilon_{y}=-7.0 \pm 2.4 \mathrm{mas} \\
\varepsilon_{z}=+16.8 \pm 2.4 \mathrm{mas} \\
\omega_{x}=-0.66 \pm 0.07 \mathrm{mas} / \text { year } \\
\omega_{y}=+0.84 \pm 0.07 \mathrm{mas} / \text { year } \\
\omega_{z}=+0.14 \pm 0.07 \mathrm{mas} / \text { year }
\end{gathered}
$$

\section{The equator and the ecliptic}

Due to precession, the celestial equator, defined as the plane perpendicular to the rotation axis of the Earth, will move freely in the new coordinate system and in the future, will tend to deviate increasingly from the fixed $X O Y$ reference plane (see Figure 1).

The ecliptic plays no role in the fundamental system. It may be useful still to give expressions for the position of the node $\bar{\gamma}$ of the ecliptic on the fixed XOY plane. It is, important to note, that it cannot be considered as an origin on the ecliptic. However, it is useful to have a fixed reference coordinate system close to the present ecliptic system for use in describing the motions in the solar system. The best choice might be that it is derived from the $O X Y Z$ axes by a rotation around $O X$ of an angle $\varepsilon_{0}$ equal to the mean obliquity of the ecliptic at J2000. This would mean that $\gamma_{0}$ is also the origin of the fixed solar system reference frame.

\section{The true equatorial reference frame}

For observations from the ground, one must use a true equatorial reference frame (TERF) defined by the orientation of the Earth in space. The system is defined by the true equator at the time of observation, perpendicular to the direction of the instantaneous axis of rotation of the Earth (the celestial pole). Another solution is to take the Celestial Ephemeris Pole. What follows does not depend upon the choice between these two poles. The position of the true equator may be defined by two angles: 
- the angle between the origin $\gamma_{0}$ of the ICRF and the ascending node of the equator, $N$,

- the inclination $i$ of the equator on the reference $X O Y$ plane.

A third angle will define the position of the origin of angles reckoned along the equator. Several definitions could be adopted for the position of this point.

Let us list five possibilities which may be proposed.

(a) The origin $\gamma(t)$ is the intersection of the moving ecliptic on the moving equator. This is the true equinox, which is not adequate because it is not defined with a precision comparable with the definition of the equator.

(b) The intersection $K$ of the moving equator with the fixed $X Z$ plane. It moves on the sky on this plane with an amplitude equal to $\varepsilon$, the obliquity of the ecliptic, with a period of 26000 years.

(c) The intersection $H$ of the moving equator with the celestial meridian defined by the celestial pole $P(t)$ at time $t$ and $\gamma_{0}$. With respect to ICRS, $H$ moves in 26000 years on a curve whose amplitude is of the order of $2 \pi(1-\cos \varepsilon)$.

(d) A point $\Sigma$ on true equator such that $\Sigma N=\gamma_{0} N$.

(e) The point $\sigma$ on the moving equator such that its instantaneous motion has no rotation component around the moving polar axis. This is the departure point (also presented as the non-rotating origin) which is such that no correction due to the system of coordinates is to be added to $d(U T) / d t$ in order to represent the absolute rate of rotation of the Earth. It is defined by

$$
Q=\sigma N-\gamma_{0} N=\frac{1}{2} \int_{t_{0}}^{t}\left(\frac{d x}{d t} y-x \frac{d y}{d t}\right) d t
$$

where $x, y$ and $z$ are, in the ICRS, the direction cosines of the vector $O P(t)$ (Capitaine et al., 1986). The quantity $Q$ can be directly computed from the expressions of precession and nutation in the fixed reference system. All other choices of the origin but the last one, introduces spurious terms in UT1.

It is to be noted that for all solutions except the first, the ecliptic plays no role in the definition of the reference frame nor in the origin of the moving celestial coordinates attached to the true celestial pole.

\section{Precession and nutation}

These quantities referred to the ICRS are now representative of a single physical phenomenon: the motion of the Earth's pole on the sky. In particular, precession is no more corrupted by the motion of the ecliptic or the rotation of the Galaxy. As a consequence, there is no longer any reason to separate them and one should produce a single set of formulae with the short (nutation), long and secular (precession) terms of the motion of $P(t)$. If $\sigma$ is chosen as the origin on the moving equator, the reference expression for $Q$ should be provided together with the nutation-precession series. These quantities describe the relation $R$ between the ICRF and the ITRF as a function of time. It would be also useful to provide consistent expressions of $\gamma_{0} N, N \sigma$ and $i$.

Let us call $\psi=\gamma_{0} N$ and $\phi=N \sigma$. Let $S$ be a star with coordinates $\alpha, \delta$ in the fixed system and $\alpha^{\prime}, \delta^{\prime}$ in the moving equatorial system with its origin in $\sigma$, the transformation from $\alpha, \delta$ to $\alpha^{\prime}, \delta^{\prime}$ is

$$
\left(\begin{array}{c}
\cos \delta^{\prime} \cos \alpha^{\prime} \\
\cos \delta^{\prime} \sin \alpha^{\prime} \\
\sin \delta^{\prime}
\end{array}\right)=R\left(\begin{array}{c}
\cos \delta \cos \alpha \\
\cos \delta \sin \alpha \\
\sin \delta
\end{array}\right)
$$

where $R$ is given by 


$$
\left(\begin{array}{ccc}
\cos \phi \cos \psi-\operatorname{cosisin} \phi \sin \psi & \cos \phi \sin \psi+\cos i \sin \phi \cos \psi & \sin i \sin \phi \\
-\sin \phi \cos \psi-\cos i \cos \phi \sin \psi & -\sin \phi \sin \psi+\cos i \cos \phi \cos \psi & \sin i \cos \phi \\
\sin i \sin \psi & -\sin i \cos \psi & \cos i
\end{array}\right)
$$

Note : if the origin is not $\sigma$, but one of the points defined in (b), (c) or (d) in Section 4, this formula does not change, provided that $\phi$ is differently defined.

\section{Sidereal time}

The definition of sidereal time will depend upon the choice that is made for the origin on the moving equator. It must be consistent with the adopted definition of UT1. If the adopted origin of UT1 is the departure point $\sigma$, the sidereal time is strictly proportional to UT1 with no more secular terms introduced into the expression of the ratio between the length of the day and 24 hours of sidereal time, and in order to avoid misinterpretation, the expression stellar angle might be preferably used. Its definition would be the hour angle of the origin $\sigma$ reckoned from the origin of terrestrial longitudes. Its derivative with respect to time is the angular velocity of the Earth around its polar axis.

\section{Stellar positions}

The positions of stars on a date $t$ reckoned from J2000 in the ICRS have to be corrected only for proper motion and parallaxes as well as, if deemed useful, the light deflection and the perspective acceleration. The observation of a star from the Earth will provide coordinates in the ITRF. Applying the ITRF-ICRS transformation matrix, one will obtain directly its position in the fixed ICRS for the epoch of observation. Any change of epoch will be made applying only the above mentioned corrections.

Remark - One of the changes with respect to the present situation is that no intermediary mean equinox of data or mean sidereal time need to be introduced. With the advent of computers, the conventional relation $R$ and its inverse will be available for the time $t$ of observation and their application straightforward to go from the true equatorial system to the International Celestial Reference System and vice-versa.

\section{Conclusions}

The adoption of the ICRS will impose some requirements on users for consistency in the implementation of precise reference frames. It will also provide an opportunity to make changes that will simplify future use of reference systems.

It will be necessary, now, for users to allow for the corrections to the offsets of the celestial pole with respect to the current IAU adopted theories of precession and nutation. The procedure to transform between a terrestrial frame and the ICRS is not consistent with the current IAU theories at the level of a milliarcsecond. Adoption of the ICRS will also require that users of frames other than Hipparcos account for significant systematic errors in the positions and motions of objects in the non-ICRS frames.

Adoption of the ICRS will also allow us to dispose of the poorly defined dynamical equinox in the definition of astronomical reference systems. A more straightforward approach will be possible in the determination of the orientation of the astronomical reference systems with respect to terrestrial systems. A much more accurate and consistent theory of precession/nutation can also be adopted as well as an improved definition of sidereal time.

\section{References}

Capitaine, N., Guinot, B. and Souchay, J. (1986) A non-rotating origin on the instantaneous equator: definition, properties ans use. Celest. Mech., 39, pp. 283-307.

ESA (1997) The Hipparcos and Tycho Catalogues. ESA Publ. SP-1200, Vol. 3.

Ma, C. and Feissel, M. (eds) (1997) IERS Technical Note, 23, Observatoire de Paris. 